\title{
Volumetric upper airway changes following a new technique for Surgically-Assisted Maxillary Expansion (SAME)
}

\section{Zmiany objętościowe górnych dróg oddechowych po zastosowaniu nowej techniki wspomaganego chirurgicznie poszerzania szczęki}

\author{
Shadi Yazigii,A-F , Mazen Zenati ${ }^{2, A-F}$, Rania Hadad ${ }^{3, A-F}$ \\ ${ }^{1}$ Department of Oral and Maxillofacial Surgery, Faculty of Dental Medicine, Damascus University, Syria \\ ${ }^{2}$ Department of Oral and Maxillofacial Surgery, Faculty of Dentistry, Syrian Private University, Damascus, Syria \\ ${ }^{3}$ Department of Orthodontics and Dentofacial Orthopedics, Faculty of Dental Medicine, Damascus University, Syria \\ A - research concept and design; $\mathrm{B}$ - collection and/or assembly of data; $\mathrm{C}$ - data analysis and interpretation; \\ $D$ - writing the article; $E$ - critical revision of the article; $F$ - final approval of the article
}

Address for correspondence

Shadi Yazigi

E-mail: shadiyazigi@gmail.com

Funding sources

None declared

Conflict of interest

None declared

Received on September 16, 2018

Reviewed on 0ctober 7, 2018

Accepted on 0ctober 23, 2018

Published online on December 31, 2018

Cite as

Yazigi S, Zenati M, Hadad R. Volumetric upper airway changes following a new technique for Surgically-Assisted Maxillary Expansion (SAME). Dent Med Probl. 2018;55(4):371-378. doi:10.17219/dmp/99158

DOI

10.17219/dmp/99158

Copyright

○ 2018 by Wroclaw Medical University

and Polish Dental Society

This is an article distributed under the terms of the

Creative Commons Attribution Non-Commercial License

(http://creativecommons.org/licenses/by-nc-nd/4.0/)

\begin{abstract}
Background. Skeletal maxillary constriction (SMC) is one of the common skeletal discrepancies which are associated with alterations in the respiratory function. Today, many surgical techniques are used to expand the maxilla in adult patients with no consensus about the optimal technique.

Objectives. The present study aimed to investigate the changes of the upper airway volume resulting from the use of a new, minimally invasive surgically-assisted maxillary expansion (SAME) technique, and compare the results with the conventional SAME technique.
\end{abstract}

Material and methods. A prospective study was conducted between September 2015 and July 2018. A total of 28 adult patients (11 males, 17 females; mean age: $19.1 \pm 2.7$ years) with SMC underwent SAME. The sample was divided according to the applied surgical technique into 2 groups: the conventional osteotomy SAME (CO-SAME) group consisted of 13 patients (18.7 \pm 2.2 years) and the selective osteotomy SAME (SO-SAME) group consisted of 15 patients ( $19.4 \pm 3.2$ years). Cone beam computed tomography (CBCT) scans were initially obtained preoperatively (T1) and 3 months post expansion (T2). The upper airway was divided into 2 segments: retropalatal and retroglossal. The volume of each segment and the total airway volume (TAV) were assessed using the 0 nDemand $3 D^{\circledR}$ software.

Results. The total upper airway volume showed a significant increase after both CO-SAME and SO-SAME $(1.29 \pm 0.26 \mathrm{cc}$ and $1.21 \pm 0.19 \mathrm{cc}$, respectively), with significant increases in retropalatal and retroglossal airway volumes (RPAV and RGAV) after both CO-SAME and SO-SAME (RPAV $-0.73 \pm 0.10 \mathrm{cc}$ and $0.83 \pm 0.10 \mathrm{cc}$, and $R G A V-0.56 \pm 0.23$ cc and $0.38 \pm 0.23$ cc, respectively). No significant differences were observed in the maxillary width (MW), TAV or RGAV between the 2 SAME techniques, whereas the increase in RPAV in the SO-SAME group was significantly greater than that of the CO-SAME group.

Conclusions. The new, minimally invasive SAME technique was an effective procedure to increase MW and the upper airway volume.

Key words: skeletal maxillary constriction, surgically-assisted maxillary expansion (SAME), upper airway volume

Słowa kluczowe: szkieletowe zwężenie szzzęki, wspomagane chirurgiczne poszerzanie szzzęki, objetoś́ górnych dróg oddechowych 


\section{Introduction}

Skeletal maxillary constriction (SMC) is a frequent component of malocclusions in non-syndromic patients. ${ }^{1}$ Clinical features of SMC include a narrow palate, unilateral or bilateral posterior cross bite, dental crowding, and difficulty in nasal breathing. ${ }^{2-4}$

Several approaches are available to treat SMC, including rapid maxillary expansion (RME) and slow maxillary expansion (SME), which are successful in correcting this discrepancy in children aged up to $13-15$ years, but they are inefficient after the ossification of the mid-palatal suture occurs. Surgically-assisted maxillary expansion (SAME) is indicated in patients who have already achieved maturity of the mid-palatal suture. ${ }^{1-3}$

Since the early $20^{\text {th }}$ century, the SAME techniques have been developed with different osteotomies. Osteotomies of the zygomatic buttresses, the separation of the pterygomaxillary junction, the separation of the mid-palatal suture, or a combination of these procedures represent the most common techniques, as they involve the most common areas that resist maxillary expansion. ${ }^{3}$

Despite the effectiveness of SAME as a treatment for $\mathrm{SMC}$, the literature provides no consensus about the minimum of osteotomies required for effective expansion. ${ }^{3}$ Nevertheless, most authors agree that there is a need for a zygomatic buttress osteotomy. ${ }^{3,5}$ However, the tendency is toward minimally invasive surgery. ${ }^{6}$

The relationship between SMC and respiratory problems, such as obstructive sleep apnea, has received increasing attention in the recent literature. ${ }^{4,7,8}$ The volume of air passing through the nose and the nasopharynx is limited by the shape and diameter of the latter. ${ }^{9}$ Some authors observed SMC in patients who presented with constricted nasopharyngeal dimensions and altered the respiratory function. ${ }^{9}$

Maxillary expansion procedures widen the nasal floor and reduce the resistance to airflow, with a positive influence on the respiratory function. ${ }^{7}$ Angell first proposed RME as a treatment for respiratory disturbances in the $19^{\text {th }}$ century. ${ }^{\text {acc. } 10}$

Today, various studies show nasal respiratory improvement after SAME and RME, and these have used a lot of methodologies to evaluate nasal airflow, such as acoustic rhinometry, ${ }^{11}$ rhinomanometry, ${ }^{11,12}$ and tomography evaluation. ${ }^{13-15}$

There are few studies in the literature that show the effect of SAME on the upper airway and compare the influence of different SAME techniques on the upper airway dimensions. ${ }^{7,16,17}$ Therefore, the aim of this study was to show the effects of a new, minimally invasive SAME technique on the upper airway dimensions, and compare the results with the conventional SAME technique using cone beam computed tomography (CBCT).

\section{Material and methods}

\section{Study design}

A prospective study was conducted between September 2015 and July 2018 at the Department of Oral and Maxillofacial Surgery and the Department of Orthodontics and Dentofacial Orthopedics, Faculty of Dental Medicine of Damascus University, Syria.

The study protocol was approved by the Ethics Committee of Damascus University at the Ministry of Higher Education of Syria (protocol No. 51). Written informed consent was obtained from each participant and/or their legal representative, as appropriate.

\section{Sample size estimation}

The sample size was calculated using the Minitab ${ }^{\circledR}$ v. 16.2.1 software (Minitab, Inc., State College, USA) in accordance to a study by Günbay et al., ${ }^{18}$ who observed an increase in the maxillary width (MW) $(5.25 \pm 1.46 \mathrm{~mm})$ after SAME, which is the most important variable that confirms maxillary expansion. The following assumptions were used: the statistical test was the sample $t$-test with a statistical power of $90 \%$ and a significance level of 0.05 . The sample size of at least 26 patients was necessary (13 patients for each group). Taking into consideration sample attrition, additional 2 patients were added to each group, which made the total sample size of 30 patients (15 patients for each group).

Ultimately, we eliminated 2 patients because of the inability to follow up throughout the observation period. Eventually, data from 28 patients were used for the statistical analysis.

\section{Patient selection}

Patients were selected from the Department of Orthodontics and Dentofacial Orthopedics at Damascus University. The study sample included 28 adult patients: 17 women $(60.7 \%)$ and 11 men (39.3\%) aged $17-28$ years (19.1 \pm 2.7 years). The inclusion criteria were as follows: adult normal healthy patient - according to the American Society of Anesthesiologists (ASA) classification, ${ }^{19}$ SMC $>5 \mathrm{~mm}$ - according to the Ricketts analysis, ${ }^{5}$ and age $>16$ years. ${ }^{5}$ The exclusion criteria were as follows: systemic disease, syndromes, maxillofacial deformities, previous maxillofacial trauma, previous orthodontic treatment, and/or previous maxillofacial surgery.

According to the surgical protocol that was undertaken, the patients were divided into 2 groups: the conventional osteotomy SAME technique (CO-SAME) group, which included 13 patients ( 9 females, 4 males; mean age: $18.7 \pm 2.2$ years), and the selective osteotomy SAME technique (SO-SAME) group, which included 15 patients ( 8 females, 7 males; mean age: $19.4 \pm 3.2$ years) . 


\section{Surgical interventions}

All surgical procedures were done under local anesthesia, 2\% lidocaine with 1/80,000 epinephrine (Kwang Myung Pharm Co., Ltd., Seoul, South Korea), and all osteotomies were accomplished with a piezo-surgical device (Implant Center; SATELEC, ACTEON Equipment, Mérignac, France) at the voltage of $100-115-230 \mathrm{~V} 50 / 60 \mathrm{~Hz}$ and ultrasonic frequency of $28-36 \mathrm{kHz}$.

In the CO-SAME group, the osteotomy technique protocol followed the methods reported by Bierenbroodspot et al. ${ }^{20}$ Briefly, a bilateral LeFort-1 osteotomy extended from the piriform rims anteriorly, through the zygomatic buttress, to the tuberosity area posteriorly, $5 \mathrm{~mm}$ above the apices of the maxillary teeth with 2 para midline palatal osteotomies (Fig. 1,2).

In the SO-SAME group, the osteotomy technique protocol followed the methods reported by de Freitas et al., ${ }^{21}$ involving bilateral zygomatic buttress osteotomies (Fig. 3). The first author of the present study added bilateral nasal buttress osteotomies (Fig. 3) and modified a palatal osteotomy by adding a $\mathrm{V}$-shaped midline osteotomy (Fig. 4) to ensure a complete mid-palatal suture separation and a full disjunction between the maxilla and the nasal septum.

The hyrax ${ }^{\circledR}$ tooth-borne expanders (DENTAURUM $\mathrm{GmbH} \&$ Co., Ispringen, Germany) were used for all the patients. After a latency period of 3 days, the patients started activating the expander twice a day as follows: 2 turns in the morning and 2 turns in the evening, corresponding to an expansion rate of $0.88 \mathrm{~mm}$ per day, until adequate expansion was achieved. At the end of the active expansion phase, the hyrax screw was stabilized for 3 months with a stainless-steel ligature wire and a flowable composite.
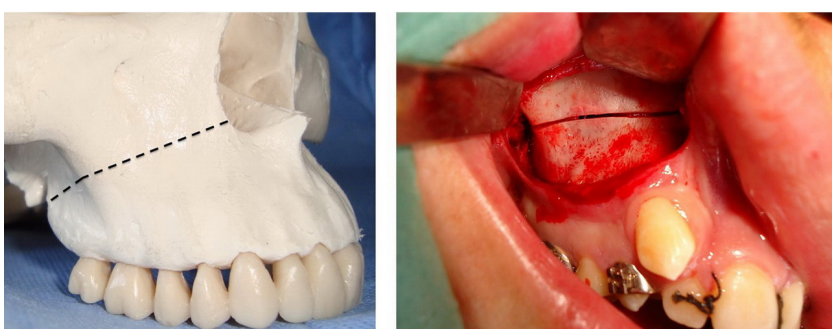

Fig. 1. LeFort-1 osteotomy $5 \mathrm{~mm}$ above the apices of the maxillary teeth
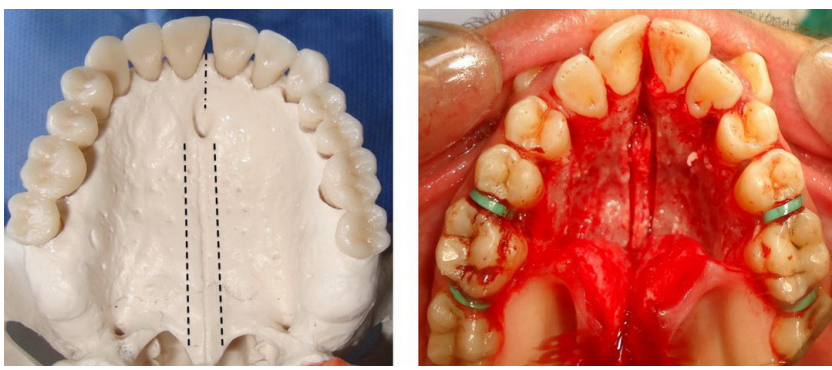

Fig. 2. Two para midline palatal osteotomies
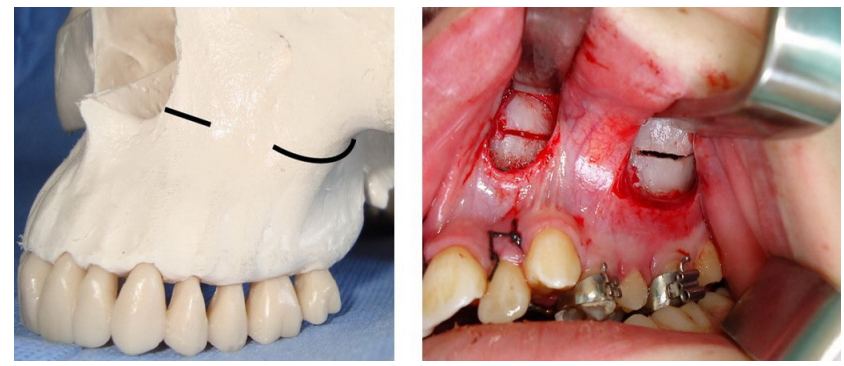

Fig. 3. Zygomatic and nasal buttress osteotomies

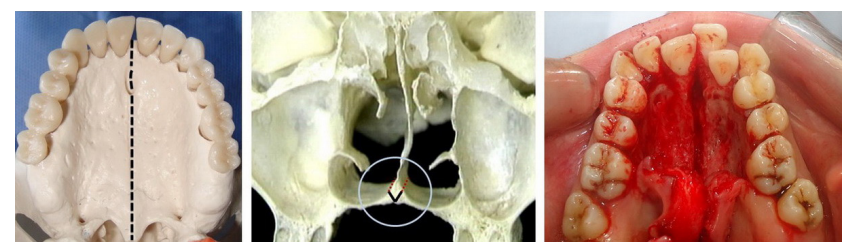

Fig. 4. V-shaped midline osteotomy

\section{Outcome measures}

Cone beam computed tomography scans were done for all the patients before the operation (T1) and 3 months after expansion (T2), and all CBCT scans were performed using the same device (SCANORA ${ }^{\circledR}$; SOREDEX, Tuusula, Finland) with $85 \mathrm{kV}, 15 \mathrm{~mA}$ and the exposure time of $2.25 \mathrm{~s}$. The field of view (FOV) was $13.5 \times 17 \mathrm{~cm}$ and the voxel size was $0.3 \mathrm{~mm}$. All the Digital Imaging and Communications in Medicine (DICOM) data was processed with the OnDemand3D ${ }^{\circledR}$ software, the 1.0.8.0408 edition (Cybermed, Inc., Tustin, USA).

Each patient was seated in a chair with their Frankfort horizontal plane parallel to the floor and asked to hold their breath after the end of expiration, without swallowing. This patient position ensures a static pharyngeal airway size, which can be recorded consistently in all CBCT scans.

For more accuracy, measurements were performed after all CBCT images were oriented to the midsagittal view (the horizontal axis passing through the anterior and posterior nasal spine - ANS and PNS) (Fig. 5) and to the axial view (the vertical axis passing through ANS and PNS) (Fig. 6).

The maxillary width was calculated and compared preand post-expansion. To calculate MW, all CBCT images were standardized at the level of the first molar furcation on the axial dimension (Fig. 7). The deepest points in the concavity of the posterior maxilla were identified on the right and left sides. The maxillary width was measured along the line connecting these 2 points (Fig. 8).

The upper airway volume was defined as the airway volume between the 2 planes: the superior plane, defined on the midsagittal slice as the horizontal line extending from PNS to the basion (called the P-plane), and the inferior plane, defined as the horizontal line passing through the most superior point of the epiglottis and parallel to the P-plane (called the EP-plane). 


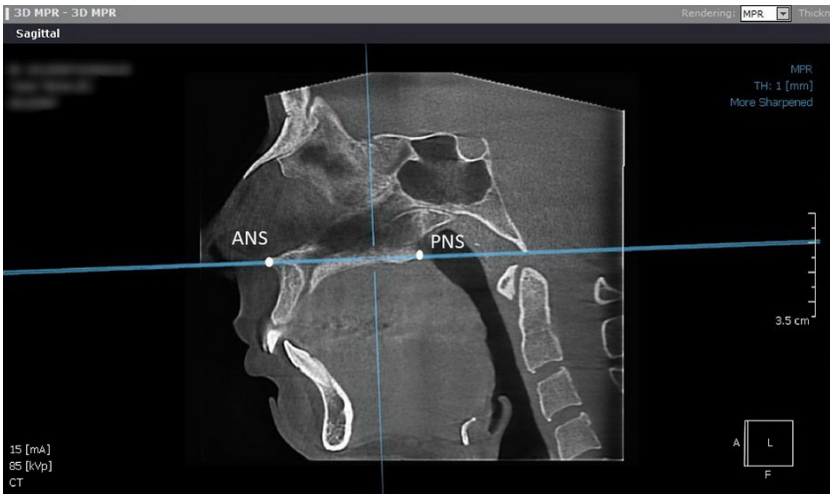

Fig. 5. Horizontal axis passing through the anterior (ANS) and posterior nasal spine (PNS)

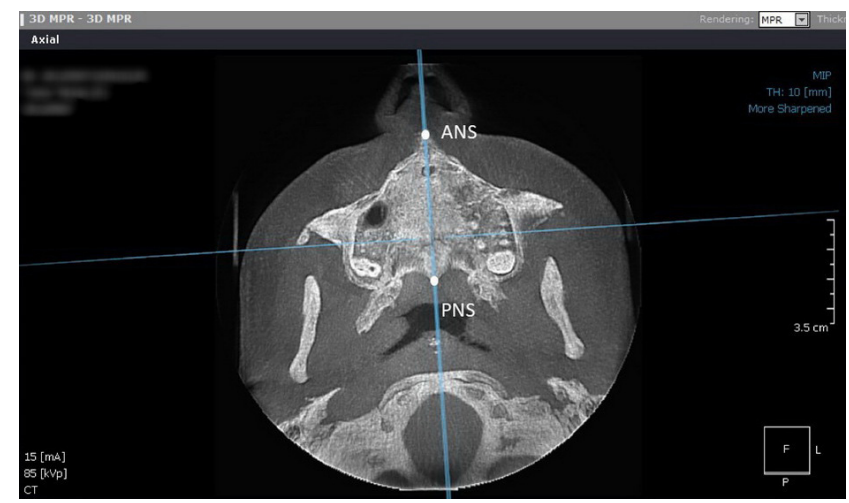

Fig. 6. Vertical axis passing through ANS and PNS

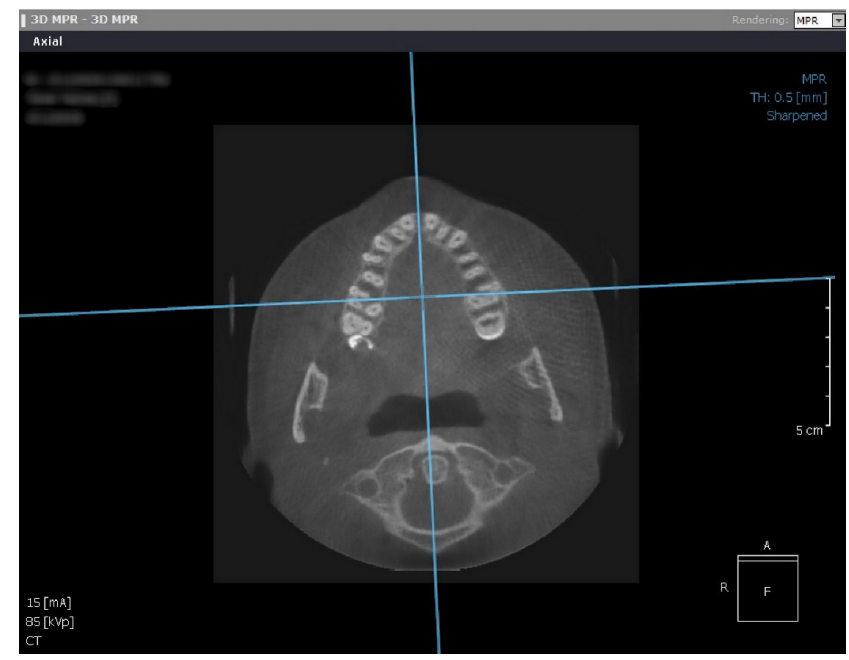

Fig. 7. Horizontal axis passing through the first molar furcation on both sides

The upper airway was divided into 2 segments to further evaluate the effects of SAME. The superior segment or retropalatal airway was limited superiorly by the P-plane and inferiorly by a horizontal plane passing through the most posteroinferior point of the soft palate and parallel to the P-plane (called the SP-plane). The inferior segment or retroglossal airway was extended from the SP-plane to the EP-plane (Fig. 9).

As a first step, we calculated total airway volume (TAV) using the OnDemand3D software. Then in the next step, we calculated retropalatal airway volume (RPAV), then we subtracted RPAV from TAV to obtain retroglossal airway volume (RGAV) (Fig. 10,11).

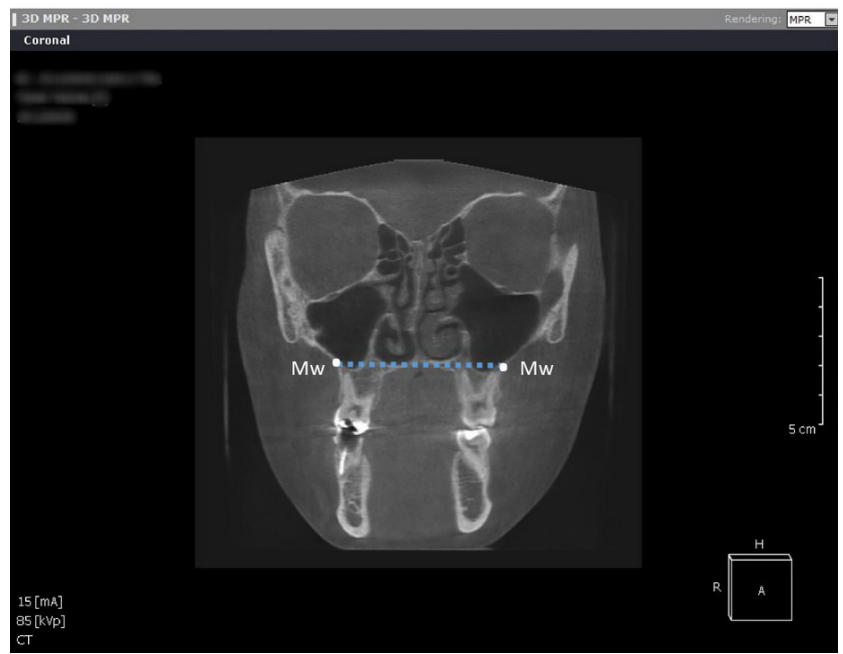

Fig. 8. Linear measurement of the maxillary width (MW)

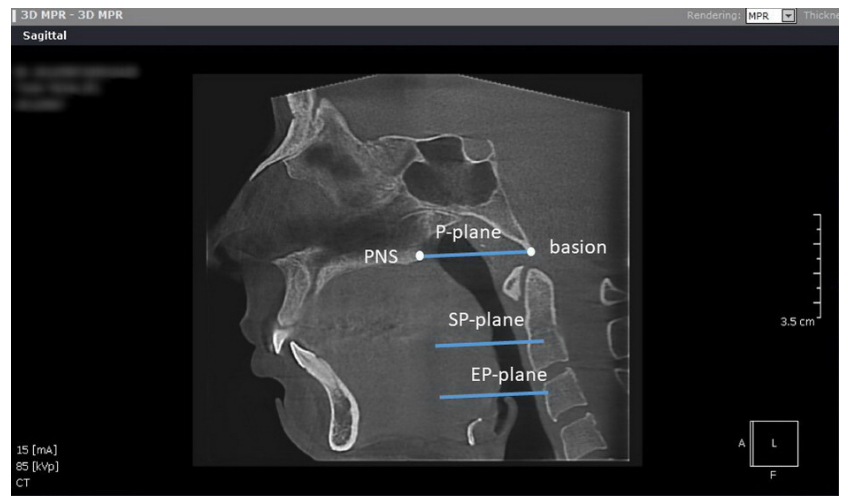

Fig. 9. Total upper airway, retropalatal and retroglossal borders

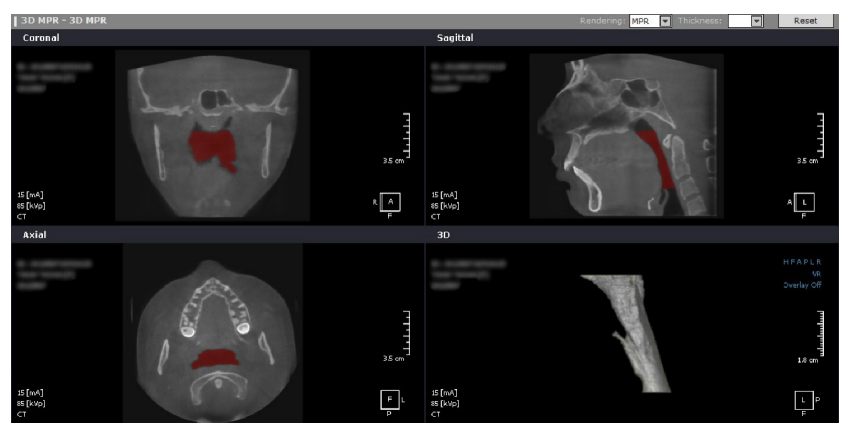

Fig. 10. Total upper airway volume (TAV)

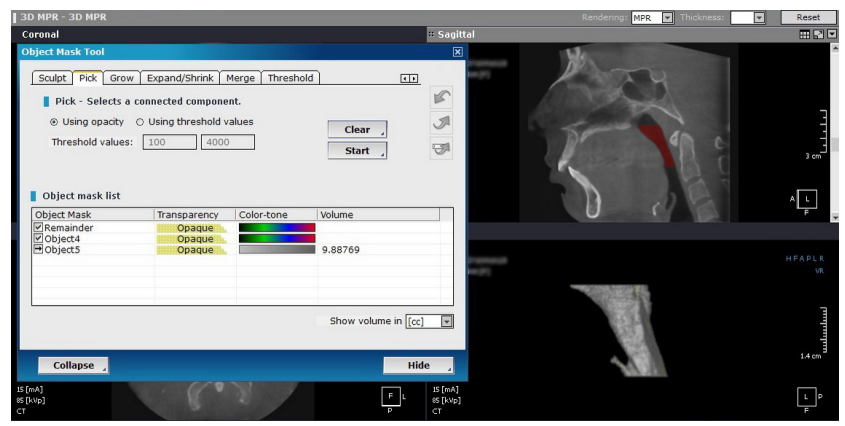

Fig. 11. Retropalatal airway volume (RPAV) 


\section{Reliability of measurements}

Ten CBCT images were randomly chosen from the sample. The landmarks were identified, and MW, TAV and RPAV were calculated. All 10 CBCT images were traced again 4 weeks later. The error of the measurements was calculated by means of the intraclass correlation coefficient (ICC) for single measurements; this is an expression of intra-observer reliability. An ICC value $>0.75$ indicates excellent reliability. The ICC values were between 0.992 and 1 (Table 1).

\section{Statistical analyses}

The SPSS v. 13.0 software for Windows (IBM Corp., Armonk, USA) was used for statistical analyses. According to the Kolmogorov-Smirnov test, the data was normally distributed. Descriptive statistical methods (mean, standard deviation - SD) and pre-/post-expansion data were compared using the sample $t$-test. The results were evaluated at the significance level of $p<0.05$ and a 95\% confidence interval (CI).

\section{Results}

Statistically significant differences between the 2 periods ( $\mathrm{T} 1$ and $\mathrm{T} 2$ ) were observed for each of the values - TAV, RPAV and RGAV in the CO-SAME group $(1.29 \pm 0.26 \mathrm{cc}, 0.73 \pm 0.10 \mathrm{cc}$ and $0.56 \pm 0.23 \mathrm{cc}$, respectively), and also in the SO-SAME group (1.21 $\pm 0.19 \mathrm{cc}$, $0.83 \pm 0.10 \mathrm{cc}$ and $0.38 \pm 0.23 \mathrm{cc}$, respectively) (Table 2 ).

The retropalatal airway volume was significantly greater than RGAV after both CO-SAME and SO-SAME (Table 3). There were no significant differences in MW $(p=0.790)$, TAV $(p=0.315)$ and $\operatorname{RGAV}(p=0.052)$ between the 2 techniques, whereas RPAV in the SO-SAME group was significantly greater than that of the CO-SAME group $(p=0.015)$ (Table 4$)$.

Table 1. The intraclass correlation coefficient (ICC) values between the values in 2 readings

\begin{tabular}{|c|c|c|c|}
\hline \multirow{2}{*}{ Second variable } & \multirow{2}{*}{ Studied variable } & First variable (values in the $1^{\text {st }}$ reading) & \multirow{2}{*}{ Reproducibility } \\
\hline & & ICC value & \\
\hline \multirow{4}{*}{ Values in the $2^{\text {nd }}$ reading } & $\mathrm{MW}[\mathrm{mm}]$ & 0.998 & Excellent \\
\hline & $\operatorname{RPAV}[\mathrm{CC}]$ & 0.992 & Excellent \\
\hline & $\operatorname{RGAV}[C \mathrm{C}]$ & 1.000 & Excellent \\
\hline & $\mathrm{TAV}[\mathrm{Cc}]$ & 0.997 & Excellent \\
\hline
\end{tabular}

ICC - intraclass correlation coefficient; MW - maxillary width; RPAV - retropalatal airway volume; RGAV - retroglossal airway volume; TAV - total airway volume.

Table 2. Paired sample Student's t-test results between the 2 studied periods (before expansion, after 3 months) according to the studied technique

\begin{tabular}{|c|c|c|c|c|c|c|c|c|c|c|}
\hline $\begin{array}{l}\text { Studied } \\
\text { variable }\end{array}$ & $\begin{array}{l}\text { Studied } \\
\text { technique }\end{array}$ & Studied period & $\mathrm{N}$ & Mean & SD & Min & Max & $\begin{array}{c}\text { Mean } \\
\text { difference }\end{array}$ & $t$-value & $p$-value \\
\hline \multirow{4}{*}{$\begin{array}{l}\mathrm{MW} \\
{[\mathrm{mm}]}\end{array}$} & \multirow{2}{*}{ SO-SAME } & before expansion & 15 & 52.89 & 2.67 & 49.70 & 57.70 & \multirow{2}{*}{4.09} & \multirow{2}{*}{36.329} & \multirow{2}{*}{$0.000^{*}$} \\
\hline & & after 3 months & 15 & 56.98 & 2.69 & 53.40 & 61.30 & & & \\
\hline & \multirow{2}{*}{ CO-SAME } & before expansion & 13 & 52.15 & 2.01 & 49.10 & 55.60 & \multirow{2}{*}{4.05} & \multirow{2}{*}{42.227} & \multirow{2}{*}{$0.000^{*}$} \\
\hline & & after 3 months & 13 & 56.19 & 1.94 & 53.00 & 59.80 & & & \\
\hline \multirow{4}{*}{$\begin{array}{l}\text { RPAV } \\
{[\mathrm{CC}]}\end{array}$} & \multirow{2}{*}{ SO-SAME } & before expansion & 15 & 9.07 & 0.78 & 7.85 & 10.23 & \multirow{2}{*}{0.83} & \multirow{2}{*}{32.903} & \multirow{2}{*}{$0.000^{*}$} \\
\hline & & after 3 months & 15 & 9.90 & 0.79 & 8.83 & 11.22 & & & \\
\hline & \multirow{2}{*}{ CO-SAME } & before expansion & 13 & 9.57 & 1.14 & 7.98 & 11.46 & \multirow{2}{*}{0.73} & \multirow{2}{*}{26.426} & \multirow{2}{*}{$0.000^{*}$} \\
\hline & & after 3 months & 13 & 10.30 & 1.14 & 8.51 & 12.13 & & & \\
\hline \multirow{4}{*}{$\begin{array}{l}\text { RGAV } \\
{[C C]}\end{array}$} & \multirow{2}{*}{ SO-SAME } & before expansion & 15 & 6.14 & 0.95 & 4.68 & 7.46 & \multirow{2}{*}{0.38} & \multirow{2}{*}{6.445} & \multirow{2}{*}{$0.000^{*}$} \\
\hline & & after 3 months & 15 & 6.52 & 0.96 & 4.90 & 7.89 & & & \\
\hline & \multirow{2}{*}{ CO-SAME } & before expansion & 13 & 5.87 & 1.19 & 4.13 & 8.88 & \multirow{2}{*}{0.56} & \multirow{2}{*}{8.611} & \multirow{2}{*}{$0.000^{*}$} \\
\hline & & after 3 months & 13 & 6.43 & 1.14 & 4.93 & 9.21 & & & \\
\hline \multirow{4}{*}{$\begin{array}{l}\text { TAV } \\
{[C C]}\end{array}$} & \multirow{2}{*}{ SO-SAME } & before expansion & 15 & 15.22 & 1.49 & 12.84 & 17.52 & \multirow{2}{*}{1.21} & \multirow{2}{*}{24.016} & \multirow{2}{*}{$0.000^{*}$} \\
\hline & & after 3 months & 15 & 16.42 & 1.50 & 13.74 & 18.62 & & & \\
\hline & \multirow{2}{*}{ CO-SAME } & before expansion & 13 & 15.44 & 1.97 & 12.88 & 20.11 & \multirow{2}{*}{1.29} & \multirow{2}{*}{18.197} & \\
\hline & & after 3 months & 13 & 16.74 & 1.99 & 13.73 & 21.16 & & & $0.000^{*}$ \\
\hline
\end{tabular}

$\mathrm{N}$ - number of patients; SD - standard deviation; SO-SAME - selective osteotomy surgically-assisted maxillary expansion; CO-SAME - conventional osteotomy surgically-assisted maxillary expansion; ${ }^{*}$ statistically significant. 
Table 3. Paired sample Student's $t$-test between the RPAV and RGAV variation according to the studied technique

\begin{tabular}{|c|c|c|c|c|c|c|c|c|c|}
\hline $\begin{array}{l}\text { Studied } \\
\text { technique }\end{array}$ & $\begin{array}{c}\text { Studied variable } \\
{[\mathrm{cc}]}\end{array}$ & $\mathrm{N}$ & Mean & SD & Min & Max & $\begin{array}{c}\text { Mean } \\
\text { difference }\end{array}$ & $t$-value & $p$-value \\
\hline \multirow{2}{*}{ SO-SAME } & RPAV variation & 15 & 0.83 & 0.10 & 0.65 & 1.03 & \multirow{2}{*}{0.45} & \multirow{2}{*}{6.087} & \multirow{2}{*}{$0.000^{*}$} \\
\hline & RGAV variation & 15 & 0.38 & 0.23 & -0.08 & 0.72 & & & \\
\hline \multirow{2}{*}{ CO-SAME } & RPAV variation & 13 & 0.73 & 0.10 & 0.53 & 0.91 & \multirow{2}{*}{0.17} & \multirow{2}{*}{2.493} & \multirow{2}{*}{$0.028^{*}$} \\
\hline & RGAV variation & 13 & 0.56 & 0.23 & 0.12 & 0.84 & & & \\
\hline
\end{tabular}

* statistically significant.

Table 4. Independent Student's t-test results between the SO-SAME technique group and the CO-SAME technique group according to the studied variable

\begin{tabular}{|c|c|c|c|c|c|c|c|c|c|}
\hline Studied variable & Studied technique & $\mathrm{N}$ & Mean & SD & Min & Max & Mean difference & $t$-value & $p$-value \\
\hline \multirow{2}{*}{$\begin{array}{c}\text { MW variation } \\
{[\mathrm{mm}]}\end{array}$} & SO-SAME & 15 & 4.09 & 0.44 & 3.60 & 5.20 & \multirow{2}{*}{0.04} & \multirow{2}{*}{0.270} & \multirow{2}{*}{0.790} \\
\hline & CO-SAME & 13 & 4.05 & 0.35 & 3.30 & 4.70 & & & \\
\hline \multirow{2}{*}{$\begin{array}{l}\text { RPAV variation } \\
\text { [CC] }\end{array}$} & SO-SAME & 15 & 0.83 & 0.10 & 0.65 & 1.03 & \multirow{2}{*}{0.10} & \multirow{2}{*}{2.606} & \multirow{2}{*}{$0.015^{*}$} \\
\hline & CO-SAME & 13 & 0.73 & 0.10 & 0.53 & 0.91 & & & \\
\hline \multirow{2}{*}{$\begin{array}{c}\text { RGAV variation } \\
{[\mathrm{CC}]}\end{array}$} & SO-SAME & 15 & 0.38 & 0.23 & -0.08 & 0.72 & \multirow{2}{*}{-0.18} & \multirow{2}{*}{-2.034} & \multirow{2}{*}{0.052} \\
\hline & CO-SAME & 13 & 0.56 & 0.23 & 0.12 & 0.84 & & & \\
\hline \multirow{2}{*}{$\begin{array}{l}\text { TAV variation } \\
{[\mathrm{cc}]}\end{array}$} & SO-SAME & 15 & 1.21 & 0.19 & 0.90 & 1.54 & \multirow{2}{*}{-0.09} & \multirow{2}{*}{-1.025} & \multirow{2}{*}{0.315} \\
\hline & CO-SAME & 13 & 1.29 & 0.26 & 0.85 & 1.76 & & & \\
\hline
\end{tabular}

* statistically significant.

\section{Discussion}

Skeletal maxillary constriction is a pathological condition that may be associated with other types of dentoskeletal alterations and can cause functional implications, including respiratory problems. ${ }^{7}$ The anatomic proximity of the pharynx to the oral cavity suggests that any change in the intraoral volume changes the oropharyngeal dimensions. ${ }^{8}$ The SAME technique, which offers the maxillary bony segment expansion, might also be effective in increasing the nasopharyngeal dimensions. ${ }^{10}$

Cone beam computed tomography is considered to be a reliable and accurate method for measuring the pharyngeal volume ${ }^{22}$ and OnDemand3D is considered to be reliable software to measure the airway volume. ${ }^{23}$

There is inconsistent data in the literature about the maxillary expansion (RME and SAME) effects on the upper airway. Pangrazio-Kulbersh et al. evaluated the effect of RME on the airway volume and concluded that the airway volume did not significantly change after expansion. ${ }^{14} \mathrm{El}$ and Palomo reported that RME significantly increased the nasal passage airway volume without any significant change in the oropharyngeal airway volume. ${ }^{15}$ Zhao et al. declared that there was no evidence supporting the hypothesis that RME could increase the oropharyngeal airway volume..$^{24}$ Fastuca et al. stated that RME significantly increased TAV. ${ }^{25}$ Iwasaki et al. observed that RME not only reduced the nasal obstruction, but also raised the tongue and enlarged the pharyngeal airway volume. ${ }^{26}$ Buck et al. presented a systematic review on the effects of surgically-assisted rapid maxillary expansion (SARME) on the upper air- way volume and concluded that the effects of SARME on the respiratory function still needed to be evaluated, ${ }^{27}$ and that was the aim of the present study.

In the present study, MW increased significantly after the application of either of the 2 SAME surgical techniques, in the SO-SAME and CO-SAME groups $(4.09 \pm 0.44 \mathrm{~mm}$ and $4.05 \pm 0.35 \mathrm{~mm}$, respectively), and there was no significant difference between the 2 techniques $(p=0.79)$. This is in accordance with Sygouros et al. and indicates the skeletal effects of the 2 SAME techniques on 2 maxillary bony segments. ${ }^{28}$

In the present study, RPAV, RGAV and TAV significantly increased after 3 months in the SO-SAME group $(0.83 \pm 0.10 \mathrm{cc}, 0.38 \pm 0.23 \mathrm{cc}$ and $1.21 \pm 0.19 \mathrm{cc}$, respectively) and in the CO-SAME group $(0.73 \pm 0.10 \mathrm{cc}, 0.56 \pm 0.23 \mathrm{cc}$ and $1.29 \pm 0.26 \mathrm{cc}$, respectively). These changes might be explained by the effect of SAME on the posterior part of the maxilla and subsequently on the palatal muscles, as well as by the anterior displacement of the maxilla and the change in the tongue position.

These results do not correlate with those of Pereira-Filho et al., who evaluated the effect of SARME on the upper airway volume and stated that there were no significant differences in TAV measured preoperatively and 6 months after expansion. ${ }^{7}$ This disagreement might result from the different surgical techniques applied by Pereira-Filho et al., which did not comprise a palatal osteotomy. ${ }^{7}$

The results of this study correlate with those of Rômulo de Medeiros et al., who applied 2 surgical techniques of SARME, with and without a pterygomaxillary disjunction. ${ }^{17}$ They assessed the volumetric changes of the nasopharynx and the oropharynx preoperatively, after the 
activation period and at 6 months after expansion. They declared that there was a significant increase in the nasopharynx and oropharynx volume immediately after the hyrax screw stabilization. Furthermore, these results are in accordance with the observations made by Vinha et al., who stated that SARME promoted the pharyngeal enlargement, especially in the lower levels of the pharynx. ${ }^{29}$

On the other hand, contrary results are presented by Kurt et al., who compared the effect of SARME on the nasopharyngeal airway using lateral and posteroanterior cephalograms. ${ }^{10}$ They concluded that no significant difference was found in the nasopharyngeal airway after SARME. This disagreement might be due to the different surgical techniques and different method of X-ray assessment.

The present study showed that the change in RPAV was greater than that in RGAV in both groups. It may result from a direct effect of both the SAME techniques on the bony segments of the maxilla, especially on the posterior part of the maxilla, where the soft palate muscles attach. The change in the position of these muscles may affect the retropalatal airway. In addition, the SAME technique improves nasal breathing, and this may reduce the thickness of the retropalatal airway respiratory mucosa.

Similar results were presented by Sadeghian et al., who concluded that the average increases in RPAV and TAV were statistically significant, while the change in RGAV was not significant. ${ }^{30}$ The change in RGAV may have resulted from the effect of the SAME technique on the tongue position, and that agrees with Akay et al., who concluded that the transpalatal distraction caused changes in the tongue height, the nasopharyngeal airway dimensions and the minimal oropharyngeal distance behind the tongue base. ${ }^{31}$

The results of the present study show that the changes in RPAV associated with the SO-SAME technique were greater than those related to the CO-SAME technique $(p=0.015)$, and that may be explained by the difference in the pattern of palatal osteotomy between the 2 techniques. This confirms the results obtained by Rômulo de Medeiros et al. ${ }^{17}$, who found that the difference in the surgical techniques of SARME resulted in different effects on the upper airway volume.

\section{Conclusions}

Both the CO-SAME and the SO-SAME techniques increased RPAV, RGAV and TAV. The SO-SAME technique increased RPAV to a greater extent than the CO-SAME technique. Both the SAME techniques increased RPAV to a greater extent than RGAV.

The new SAME technique with a minimally invasive surgery is an effective way to increase MW and the upper airway volume. However, additional studies with longer follow-up periods may be needed to thoroughly evaluate the effectiveness of the abovementioned technique.

\section{References}

1. Daif ET. Segment tilting associated with surgically assisted rapid maxillary expansion. Int J Oral Maxillofac Surg. 2014;43:311-315.

2. Kayalar E, Schauseil M, Kuvat SV, Emekli U, Firatli S. Comparison of tooth-borne and hybrid devices in surgically assisted rapid maxillary expansion: A randomized clinical cone-beam computed tomography study. J Craniomaxillofac Surg. 2016;44:285-293.

3. Oliveira TF, Pereira-Filho VA, Gabrielli MA, Goncales ES, SantosPinto A. Effects of lateral osteotomy on surgically assisted rapid maxillary expansion. Int J Oral Maxillofac Surg. 2016;45:490-496.

4. Menegat F, Monnazzi MS, Silva BN, de Moraes M, Gabrielli MA, Pereira-Filho VA. Assessment of nasal obstruction symptoms using the NOSE scale after surgically assisted rapid maxillary expansion. Int J Oral Maxillofac Surg. 2015;44:1346-1350.

5. Betts NJ. Surgically assisted maxillary expansion. Atlas Oral Maxillofac Surg Clin North Am. 2016;24:67-77.

6. Koudstaal MJ, Poort LJ, van der Wal KG, Wolvius EB, Prahl-Andersen B, Schulten AJ. Surgically assisted rapid maxillary expansion (SARME): A review of the literature. Int J Oral Maxillofac Surg. 2005;34:709-714.

7. Pereira-Filho VA, Monnazzi MS, Gabrielli MA, et al. Volumetric upper airway assessment in patients with transverse maxillary deficiency after surgically assisted rapid maxillary expansion. Int $J$ Oral Maxillofac Surg. 2014;43:581-586.

8. Vinha PP, Faria AC, Xavier SP, Christino M, de Mello-Filho FV. Enlargement of the pharynx resulting from surgically assisted rapid maxillary expansion. J Oral Maxillofac Surg. 2016;74:369-379.

9. Gungor AY, Turkkahraman H. Effects of airway problems on maxillary growth: A review. Europ J Dent. 2009;3:250-254.

10. Kurt G, Altug-Atac AT, Atac MS, Karasu HA. Changes in nasopharyngeal airway following orthopedic and surgically assisted rapid maxillary expansion. J Craniofac Surg. 2010;21:312-317.

11. Zambon CE, Ceccheti MM, Utumi ER, et al. Orthodontic measurements and nasal respiratory function after surgically assisted rapid maxillary expansion: An acoustic rhinometry and rhinomanometry study. Int J Oral Maxillofac Surg. 2012;41:1120-1126.

12. Magnusson A, Bjerklin K, Nilsson P, Jonsson F, Marcusson A. Nasal cavity size, airway resistance, and subjective sensation after surgically assisted rapid maxillary expansion: A prospective longitudinal study. Am J Orthod Dentofacial Orthop. 2011;140:641-651.

13. Deeb W, Hansen L, Hotan T, Hietschold V, Harzer W, Tausche E. Changes in nasal volume after surgically assisted bone-borne rapid maxillary expansion. Am J Orthod Dentofacial Orthop. 2010;137:782-789.

14. Pangrazio-Kulbersh V, Wine P, Haughey M, Pajtas B, Kaczynski R. Cone beam computed tomography evaluation of changes in the naso-maxillary complex associated with two types of maxillary expanders. Angle Orthod. 2012;82:448-457.

15. El H, Palomo JM. Three-dimensional evaluation of upper airway following rapid maxillary expansion: A CBCT study. Angle Orthod. 2014;84:265-273.

16. Nada RM, van Loon B, Schols JG, et al. Volumetric changes of the nose and nasal airway 2 years after tooth-borne and bone-borne surgically assisted rapid maxillary expansion. Europ J Oral Sci. 2013;121:450-456.

17. Rômulo de Medeiros J, Ferraro Bezerra M, Gurgel Costa FW, Pinheiro Bezerra T, de Araújo Alencar CR, Studart Soares EC. Does pterygomaxillary disjunction in surgically assisted rapid maxillary expansion influence upper airway volume? A prospective study using dolphin imaging 3D. Int Oral Maxillofac Surg. 2017;46:1094-1101.

18. Günbay T, Akay MC, Günbay S, Aras A, Koyuncu BO, Sezer B. Transpalatal distraction using bone-borne distractor: Clinical observations and dental and skeletal changes. J Oral Maxillofac Surg. 2008;66:2503-2514.

19. Stronczek MJ. Determining the appropriate oral surgery anesthesia modality, setting, and team. Oral and Maxillofac Surg Clin North Am. 2013;25:357-366.

20. Bierenbroodspot F, Wering PC, Kuijpers-Jagtman AM, Stoelinga PJ. Surgically assisted rapid maxillary expansion: A retrospective study [in Dutch]. Ned Tijdschr Tandheelkd. 2002; 109(8):299-302.

21. de Freitas RR, Goncalves AJ, Moniz NJ, Maciel FA. Surgically assisted maxillary expansion in adults: Prospective study. Int J Oral Maxillofac Surg. 2008;37:797-804. 
22. Tsolakis IA, Venkat $D$, Hans MG, Alonso A, Palomo JM. When static meets dynamic: Comparing cone-beam computed tomography and acoustic reflection for upper airway analysis. Am J Orthod Dentofacial Orthop. 2016;150:643-650.

23. Chen $\mathrm{H}$, van Eijnatten $\mathrm{M}$, Wolff $\mathrm{J}$, et al. Reliability and accuracy of three imaging software packages used for 3D analysis of the upper airway on cone beam computed tomography images. Dentomaxillofac Radiol. 2017;46:20170043.

24. Zhao Y, Nguyen M, Gohl E, Mah JK, Sameshima G, Enciso R. Oropharyngeal airway changes after rapid palatal expansion evaluated with cone-beam computed tomography. Am J Orthod Dentofacial Orthop. 2010;137(Suppl 4):S71-78.

25. Fastuca R, Meneghel M, Zecca PA, et al. Multimodal airway evaluation in growing patients after rapid maxillary expansion. Europ $J$ Paediatr Dent. 2015;16:129-134.

26. Iwasaki T, Saitoh I, Takemoto Y, et al. Tongue posture improvement and pharyngeal airway enlargement as secondary effects of rapid maxillary expansion: A cone-beam computed tomography study. Am J Orthod Dentofacial Orthop. 2013;143:235-245.

27. Buck LM, Dalci O, Darendeliler MA, Papadopoulou AK. Effect of surgically assisted rapid maxillary expansion on upper airway volume: A systematic review. J Oral Maxillofac Surg. 2016;74:1025-1043.

28. Sygouros A, Motro M, Ugurlu F, Acar A. Surgically assisted rapid maxillary expansion: Cone-beam computed tomography evaluation of different surgical techniques and their effects on the maxillary dentoskeletal complex. Am J Orthod Dentofacial Orthop. 2014;146:748-757.

29. Vinha PP, Eckeli AL, Faria AC, Xavier SP, de Mello-Filho FV. Effects of surgically assisted rapid maxillary expansion on obstructive sleep apnea and daytime sleepiness. Sleep Breath. 2016;20:501-508.

30. Sadeghian S, Ghafari R, Feizbakhsh M, Dadgar S. Dimensional changes of upper airway after rapid maxillary expansion evaluated with cone beam computed tomography. Orthodont Waves. 2016:75:10-17.

31. Akay MC, Aras I, Günbay T, Aras A. Does transpalatal distraction affect pharyngeal airway dimensions and related soft tissues? J Oral Maxillofac Surg. 2014;72:1559-1564. 